\title{
Attitudes toward early detection of infection by the AIDS retrovirus among persons at high and low risk
}

\author{
EDMUND FANTINO, DAVID CASE, STEPHANIE STOLARZ-FANTINO, \\ PHYLLIS SPECHKO, and J. ALLEN MCCUTCHAN \\ University of California, San Diego, La Jolla, California
}

\begin{abstract}
Several months prior to the availability of a now-common blood test, homosexual volunteers, in a longitudinal study of their health, and typical college students were questioned about their attitudes toward early detection of acquired immunodeficiency syndrome (AIDS) and toward receiving information about desirable or fear-worthy hypothetical events. Generally, the homosexual volunteers were more interested in receiving information than were the students. Most homosexual volunteers desired human immunodeficiency virus (HIV) antibody test results, which could indicate infection, and indicated that receiving them would affect their behavior. However, all the subjects showed a preference for information on most questions, especially when the information could be used in guiding behavior change and was not virtually certain to be unfavorable. We are unaware of controlled evidence assessing whether or how knowledge of infection influences the imminent health risk of people who are asymptomatic, but prior to learning their HIV status, the homosexual volunteers who at the time had antibody in their sera were less concerned in knowing the test results of hypothetical future sexual partners than those without antibody.
\end{abstract}

This study assesses attitudes toward the early detection of infection by the retrovirus that causes acquired immunodeficiency syndrome (AIDS) among persons at high and low risk for infection. Although little is known about the impact of human immunodeficiency virus (HIV) antibody test results on subsequent health risk and sexual behavior (but see Coates et al., 1988), a number of studies have explored preferences for information about other personally fearful or unwelcome events (Case \& Fantino, 1981; Fantino \& Case, 1983; Leventhal \& Hirschman, 1982). For example, patients have admitted taking longer to seek help after discovering a symptom if they imagined possible severe consequences of their illness (Safer, Tharps, Jackson, \& Leventhal, 1979). Children of different ages responded to get information only when the information predicted their receiving rewarding outcomes (Fantino, Case, \& Altus, 1983), which is a behavior also observed in nonhuman subjects (Case \& Fantino, 1981). The children preferred a totally uninformative to an informative stimulus about nonrewarding outcomes. "Bad news" was also avoided by college students, unless it could be utilized to improve one's circumstances (Case, Fantino, \& Wixted, 1985; Fantino

\footnotetext{
The research and preparation of this manuscript were supported by NIMH Grant MH-20752 and NSF Grants BNS 83-02963 and BNS 9108719 to the University of California at San Diego (E.F., principal investigator) and by University of California, San Diego, Project 84-481 and NIH Grant HL-32471 to the University of California, San Diego (J.A.M., principal investigator). Portions of these data were presented by D. Case at the First International Conference on AIDS at Atlanta, GA, April 1985. Address correspondence to E. Fantino, Department of Psychology, 0109, University of California, San Diego, La Jolla, CA 92093-0109.
}

\& Case, 1983). We compared attitudes in high-risk homosexual volunteers in a longitudinal study of AIDS and lowrisk undergraduate students at the University of California, San Diego (UCSD), using comparable questionnaires concerning their attitudes toward receiving information about their personal contraction of aids, their AIDS antibody status, and other hypothetical good and bad news.

\section{METHOD}

\begin{abstract}
Two groups of subjects participated. The 147 subjects in Group 1 were volunteers in a longitudinal study of homosexual men at risk of AIDS, ranging in age from 20 to 72 years $(M=36)$. The distribution of homosexual volunteers in a clinical and immunological classification was $5 \%$ Centers for Disease Control (CDC)/AIDS; 3\% CDC/AIDS-related complex (ARC); $50 \%$ only one ARC sign or symptom; $24 \%$ one or more asymptomatic immune abnormalities; and $18 \%$ immunologically normal with no sign or symptoms. Volunteers learned of the study through word of mouth and were not recruited actively by the research staff. They were generally well informed about AIDS and completed their questionnaires following a lecture about AIDS by one of the authors (J.A.M.). Group 2 comprised 201 UCSD undergraduate students ranging in age from 16 to 27 years $(M=20)$. Forty-three percent of this group was male, $57 \%$ female. These subjects volunteered for the study to satisfy requirements for a psychology course. Both groups completed an "attitudes" questionnaire evaluating their attitudes toward receiving information about hypothetical favorable and unfavorable events of personal relevance as a function of the amount of advance notice and of the extent to which the information could be utilized. Two of the four sets of questions concerned AIDS and two involved other circumstances; order was balanced across subjects. These questions appear in Appendix A. In addition to this questionnaire, Group 1 volunteers were asked several questions that probed their comprehension of the presentation on AIDS, and inquired about their current health and perceived risk of developing AIDS. Additional questions regarding their feelings about receiving HIV test results and the possible effects of such results on their behavior appear in Appendix B. The questionnaires were administered several months prior to when HIV testing was first becoming available.
\end{abstract}




\section{RESULTS}

The results from the attitudes questionnaire that was given to Groups 1 and 2 are presented in Appendix A. Although the majority of participants expressed a preference for receiving information (i.e., made "yes" responses) for all questions other than $3 a$ and $4 b$, the results revealed some significant differences both within groups of subjects (as a function of delay and usefulness of information) and between the two groups of subjects.

\section{Within-Group Effects}

For the factor of delay in receiving unpredictable information (Question 1), the tendency to request information declined with increasing delay following testing. However, it was significant only for Group 2 (Group 1, Cochran's $Q=2.0, p>.1$; Group 2, $Q=25, p<.01$ ). In contrast, for the issue of delay in learning one has already been infected (Question 2), fewer subjects indicated a preference for immediate knowledge the more delayed the inevitable bad news. This was true for both groups (Group 1, $Q=13$; Group 2, $Q=45 ; p<.01$ for both). In addition, for both Questions 3 and 4, members of both groups were significantly less likely to want information when they would not be able to act upon it (McNemar's test for Questions 3a vs. 3b, for Group 1, $\chi^{2}=20$, for Group 2, $\chi^{2}=18$; for questions $4 \mathrm{a}$ vs. $4 \mathrm{~b}$, for Group 1, $\chi^{2}=34$, for Group 2, $\chi^{2}=75 ; p<.01$ for all tests).

\section{Between-Group Effects}

Differences in percentages of "yes" responses between Groups 1 and 2 appear in Appendix A. Except for Questions 1 and 4a, the Group 1 subjects judged themselves as more likely to request information. In addition, for Question 1a, there was a significant sex difference within Group 2; that is, $97 \%$ of the females responded "yes," whereas $91 \%$ of the males responded "yes" $\left(\chi^{2}=4.1\right.$, $p<.05$ ). (At the time of data collection, females were considered at relatively low risk for AIDS.)

Results from questions asked only of Group 1 (AIDS project volunteers) appear in Appendix B. In general, these results show a desire for blood test results in the event of being tested and a belief that receiving test results would have an effect on their behavior.

Subsequent to attitudes assessment, HIV antibody was measured in the homosexual volunteers by enzyme-linked immuno-sorbant assay (ELISA) and $43 \%$ were found to be positive. Those found to be negative had indicated that they were more likely to ask current or future sexual partners about antibody status than those found to be positive (82\% vs. $59 \% ; \chi^{2}=9.3, p<.01$ ). Specifically, they had indicated whether they agreed with the statement, "If I were told my HTLV-III antibody test were negative, I would want to know about this test result in my current or future sexual partners." These same subjects were also significantly more likely to feel more secure about their health upon receiving a negative result $(92 \%$ vs. $79 \%$; $\chi^{2}=4.5, p<.05$ ) and were more likely to want to know the test results if the results were available immediately
(94\% vs. $84 \%$ ), though this result missed significance $\left(\chi^{2}=3.7, p<.06\right)$. Finally, the subjects with negative antibody test scores were significantly less likely to want to know that they had won $\$ 3$ million in a lottery (Question 3a, Appendix A, $52 \%$ vs. $69 \% ; \chi^{2}=4.2, p<.05$ ).

\section{DISCUSSION}

Most of the subjects in the present study claimed that they would want to know if they had been infected with the AIDS retrovirus. Although studies suggest that fewer subjects would request unfavorable information if it were actually offered, even information certain to be bad is generally preferred over uncertainty if it can effectively guide action (e.g., Case et al., 1985; Case, Ploog, \& Fantino, 1990). The present subjects' responses are consistent with this view. Those who were told that they were hypothetically infected with the AIDS retrovirus said that they would inform their partners and practice more "safe" sex. The subjects were also much more likely to want information about events not involving AIDS when they could modify their behavior as a result of the information (Questions 3a vs. 3b and $4 a$ vs. $4 b$ ).

Homosexual subjects who were found to have negative HIV antibody tests were possibly more careful about sex than those who were positive. They were more concerned with knowing the antibody test results of future sexual partners and more likely to interpret (hypothetical) negative results as indicating greater health security. This latter difference possibly means that these careful subjects are more likely to expect and give credence to a negative result. Similarly, such subjects might be more willing to confront the results of the test (Question 1a, Appen$\operatorname{dix} A$ ) and be less likely to express interest in lottery winnings (Question 3a, Appendix A). These results are consistent with conservative versus promiscuous sexual habits.

Questionnaire results often reveal inconsistencies and the present ones are no exception. For example, $20 \%$ of the homosexual volunteers said that they would want to know the antibody test scores "under no circumstances I can think of." Yet $90 \%$ of the same subjects, in a different part of the same question, had just said that they would want to know the scores, "even if I could do nothing more than follow advice I have already been given to reduce the risk of becoming ill." Thus, at least $10 \%$ of the subjects were apparently inconsistent across these two nearly adjacent questions. Also, all the homosexual volunteers ultimately consented to having their blood tested.

With the exception of Question 1, a statistically equal or greater percentage of the higher risk Group 1 volunteers preferred to be informed compared with the lower risk Group 2 subjects. This may have been because the Group 1 subjects were already highly interested in AIDS, as reflected by their participation in a longitudinal study of the disease. The Group 2 subjects, on the other hand, probably did not believe themselves at risk and the possibility of their blood actually being tested was remote. In view of this potential bias for information on the part of Group 1, how might the significantly higher percentage of affirmative responses by the Group 2 subjects on Question 1a be explained? Very likely, the college students, especially females, would want the results because it is highly likely that they would test negative; thus, they would be receiving "'good news," which behavioral studies have shown to be highly rewarding (e.g., Case et al., 1990; Fantino \& Case, 1983). The significantly decreasing trend across Questions 1a-1c for these subjects suggests that the more delayed the good news, the less rewarding. The Group 1 participants, too, may have wanted the test results because of the possibility of a negative result; such "good news" would be sharply rewarding when compared with the chronic anxiety about AIDS that many of the homosexual volunteers experienced. For this group, however, the possibility of a positive test is also relatively likely.

Finding out that one has AIDS can scarcely be regarded as rewarding. It is surprising that the subjects overwhelmingly wanted to know, even if they could delay finding out. It is possible that these percentages are so high because the information was not certain to be unfavorable and because the questions were hypothetical; that is, the subjects were not in fact about to be told that they had AIDS. It is also likely that the subjects wanting to know if they had been infected or had AIDS intended to modify their behavior on the basis of such knowledge, as suggested earlier, for example, by informing sexual partners or by making 
preparations for the future. Indeed, $90 \%$ of the respondents believed in the possibility of reducing the risk of developing AIDS "even after acquiring an ARV infection" (Question 5, Appendix B), and in our clinical observations patients have displayed apparent signs of relief when informed that they have AIDS after long prodromal periods. However, there is a decreasing tendency to want to know one actually has AIDS if the knowledge would not otherwise be forthcoming for some time (Question 2, Appendix A).

In the same month that the present questionnaires were completed (November 1984), a group of 754 gay men in the San Francisco area were asked a comparable question about their hypothetical preference for HIV testing (McKusick et al., 1985). Compared with the establishment of the present pool of volunteers, this group's responses were actively solicited by researchers visiting public places known to be frequented predominantly by gay men. Most importantly, this study was not an intensive health-monitoring investigation, and so the eventual testing of sera was entirely self-initiated. The item read, "If a blood test was available to you free of charge for (HIV) antibodies, would you take it?"' While $72 \%$ of valid completions indicated agreement (which is understandably somewhat less than the present $80 \%-90 \%$ preference for antibody test results), $41 \%$ had actually attained their status approximately 1.5 years following the availability of free anonymous screening in California (i.e., by November 1986; Coates, Morin, \& McKusick, 1987; T. J. Coates \& L. Pollack, 1993, personal communication). Thus, at least $43 \%$ of those answering positively when the question was hypothetical (i.e., $72 \%-41 \% / 72 \%$ ), did not follow through by this point. By 1989 , or over 4 years since the initial query, $56 \%$ had been tested (Kegeles, Coates, Lo, \& Catania, 1989), representing a 56\%/72\% = $78 \%$ average rate of attitude-act correspondence.

The present results are generally consistent with the conditioned reinforcement hypothesis, which states that information is preferred according to its correlation with reinforcement (Case \& Fantino, 1981; Case et al., 1990). Thus, an individual would seek information about positive events such as winning a lottery because of its predictiveness about monetary reinforcement. On the other hand, one would wish to learn about negative events, such as being antibody positive, because of the anticipated utility of the information in improving one's circumstances. A prediction of the conditioned reinforcement hypothesis that distinguishes it from other accounts of preference for information is that "news" certain to be "bad" should be avoided, if subjects can do nothing about it. Most of the subjects in the present study responded in a manner consistent with this prediction (Question $4 \mathrm{~b}$ ).

From an epidemiological perspective, the results are not entirely encouraging despite the desire for information among homosexual men. For example, $24 \%$ of the homosexual volunteers reported that they would not warn future sexual partners about their own positive antibody test results. In addition, as many as $20 \%$ reported not wanting to know the blood test results in any case. Similarly, less than half reported that they would notify past sexual partners (thereby increasing the chance that the latter individuals might infect others). Moreover, these results are based on answers given in the presence of medical and psychological professionals. It is likely that the results would be even less encouraging in a more neutral context having less "experimenter demand." More important, however well intentioned the responders, there is a gulf between intentions and actions. Although the subjects may have every intention of practicing safer sex, of wanting to know test results, and of in- forming partners, it might be less likely in practice. Some findings suggest that individuals who learn that they are HIV-positive do report greater behavioral changes in the direction of safer sex than those who are either HIV-negative or uninformed (see Coates et al., 1988, for a review).

In summary, the subjects in most cases reported a preference for information. We have speculated that these preferences may, in part, reflect the demand characteristics of the questions, in some cases the potential for reducing anxiety and/or obtaining "good news" and, in most cases, the presumed utility of the information. For example, learning that one has either a positive or negative antibody test score could suggest differential changes in sexual practices (those with positive tests may want to inform sexual partners and may practice safer sex; those with negative tests may want sexual partners who also have negative test results) and differential life decisions. In fact, of course, the knowledge obtained may influence intentions, but not behavior.

\section{REFERENCES}

Case, D. A., \& Fantino, E. (1981). The delay-reduction hypothesis of conditioned reinforcement and punishment: Observing behavior. Journal of the Experimental Analysis of Behavior, 35, 93-108.

CASE, D. A., FANTINo, E., \& WixTED, J. (1985). Human observing: Maintained by negative informative stimuli only if correlated with improvement in response efficiency. Journal of the Experimental Analysis of Behavior, 43, 289-306.

CASE, D. A., Ploog, B. O., \& Fantino, E. (1990). Observing behavior in a computer game. Journal of the Experimental Analysis of Behavior, 54, 185-199.

Contes, T. J., Morin, S. F., \& McKusick, L. (1987). Behavioral consequences of AIDS antibody testing among gay men. Journal of the American Medical Association, 258, 1889.

Coates, T. J., Stall, R. D., Kegeles, S. M., Lo, B., Morin, S. F., \& McKusICK, L. (1988). AIDS antibody testing: Will it stop the AIDS epidemic? Will it help people infected with HIV? American Psychologist, 43, 859-864.

Fantino, E., \& CASE, D. A. (1983). Human observing: Maintained by stimuli correlated with reinforcement but not extinction. Journal of the Experimental Analysis of Behavior, 40, 193-210.

Fantino, E., CaSE, D. A., \& Altus, D. (1983). Observing rewardinformative and -uninformative stimuli by normal children of different ages. Journal of Experimental Child Psychology, 36, 437-452.

Kegeles, S. M., Coates, T. J., Lo, B., \& Catania, J. A. (1989). Mandatory reporting of HIV testing would deter men from being tested. Journal of the American Medical Association, 261, 1275-1276.

LeVenthal, H., \& Hirschman, R. S. (1982). Social psychology and prevention. In G. S. Sanders \& J. Suls (Eds.), Social psychology of health and illness (pp. 183-226). Hillsdale, NJ: Erlbaum.

McKusick, L., Contes, T., Stahl, R., Saika, G., Conant, M., \& WILEY, J. (1985, April). Stability and change in gay sex: The case of San Francisco. Paper presented at the First International Conference on AIDS, Atlanta, GA.

SAfer, M. A., Tharps, Q. J., Jackson, T. C., \& Leventhal, H. (1979). Determinants of three stages of delay in seeking care at a medical clinic. Medical Care, 17, 11-29.

APPENDIX A

Attitudes Questionnaire and Results for Groups 1 and 2

\begin{tabular}{|c|c|c|c|}
\hline \multirow[b]{2}{*}{ Questions } & \multicolumn{2}{|c|}{$\begin{array}{c}\text { Percent Yes } \\
\text { Responses }\end{array}$} & \multirow{2}{*}{$\begin{array}{c}\chi^{2} \\
\text { Between-Group } \\
\text { Differences }\end{array}$} \\
\hline & Group 1 & Group 2 & \\
\hline
\end{tabular}

1. If you did take the blood test (which indicates whether you have been exposed to the AIDS virus but not necessarily whether the disease will develop), would you like to know results:

a. Assuming the results were available immediately?

b. Assuming the results were available in 3 months?

90

89
96

92
$4.5 *$

0.6 n.s. 
APPENDIX A (continued)

Attitudes Questionnaire and Results for Groups 1 and 2

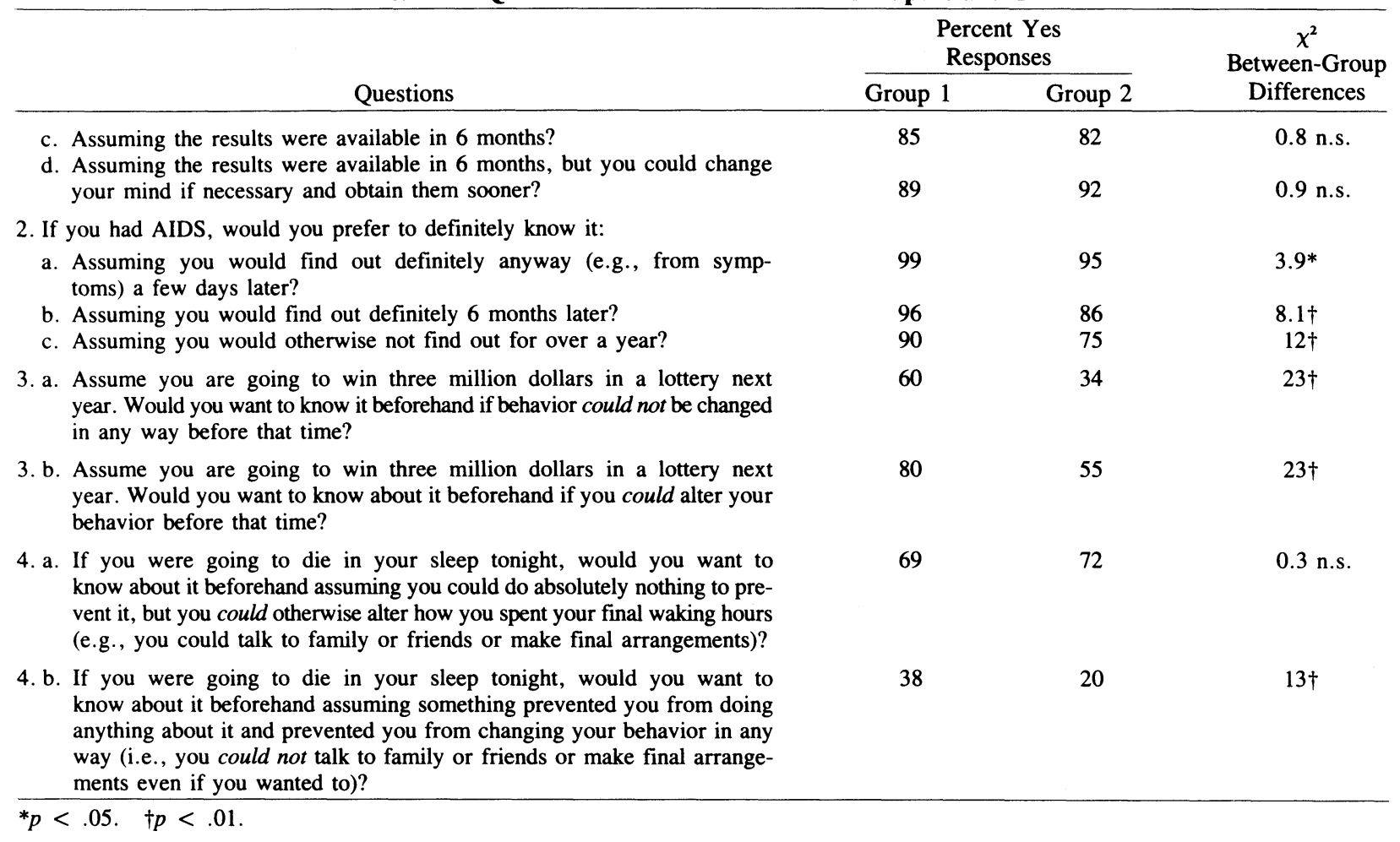

APPENDIX B

Questions Asked Only of Group 1 (Homosexual) Volunteers

Questions

Percent Yes Responses

1. If I were tested for antibody to HTLV-III, I would want the results (positive or negative):

a. Even if the test used to determine my infection could be wrong.

of becoming ill.

c. Even if I would not change my behavior in any way.

d. Under no circumstances I can think of.

2. If I were told my HTLV-III antibody test was positive, I would:

a. Modify my sexual behavior toward more "safe" sex.

b. Notify my past sexual partners.

c. Tell potential sexual partners.

d. Become more anxious about my health.

e. Not be affected in any way.

3. If I were told my HTLV-III antibody test was negative, I would:

a. Modify my sexual behavior toward more "safe" sex.

b. Want to know about this test result in my current or future sexual partners.

c. Feel more secure about my health.

d. Not be affected in any way.

4. Because medical researchers are not completely sure what a positive antibody for HTLV-III means, I would:

a. Be willing to participate in a research study in which I might be told the result of my HTLV-III antibody test or not be told in accordance with the plan of the study.

b. Not care if the test was performed, but not want to know the answer.

c. Not want the test performed on my serum.

5. Even after acquiring an ARV infection, a person might be able to reduce his risk of contracting AIDS or AIDS-related disease. 85 (1) 